\title{
XLVII. Investigation of certain remarkable and unexplained phœnomena of vision, in which they are traced to functional actions of the brain
}

\section{Mr. Thomas Smith}

To cite this article: Mr. Thomas Smith (1832) XLVII. Investigation of certain remarkable and unexplained phœnomena of vision, in which they are traced to functional actions of the brain , Philosophical Magazine Series 3, 1:4, 249-258, DOI: 10.1080/14786443208647887

To link to this article: http://dx.doi.org/10.1080/14786443208647887

曲 Published online: 01 Jun 2009.

Submit your article to this journal $\pi$

Џll Article views: 2

Q View related articles $₫$ 


\title{
LONDON AND EDINBURGH
}

\section{PHILOSOPHICAL MAGAZINE}

\author{
A N D \\ JOURNAL OF SCIENCE. \\ [THIRD SERIES.] \\ $O C T O B E R 1832$.
}

XLVII. Investigation of certain remarkable and unexplained Phcenomena of Vision, in which they are traced to Functional Actions of the Brain. By Mr. Thomas Smith, Surgeon, Fochabers*.

SOME years ago I published in this Journal $\dagger$ an account of a remarkable affection of sight which $I$ had discovered accidentally while engaged in a course of experiments on the apparent luminousness of objects, and which was thought the more worthy of being recorded, that it could be easily produced in others as well as in myself, by placing the two eyes in certain relations to light.

My former communication having been limited to a mere announcement of the new facts I had observed, I propose, in this paper, to state the results of the attempts which $I$ have made, since that period, to discover the causes of the phænomena. These results might be mentioned in a few words; but as the subject is, in almost all its bearings and relations; entirely new, and as the conclusions to which 1 have been led, tend, if confirmed, to throw a very unexpected light on the nature and exciting causes of cerebral function, of the laws of which so little is known, I have thought it incumbent on me to give each particular step of the investigation in detail, to enable the reader who takes an interest in the subject, to judge how far the conclusions are borne out by the premises. Our total ignorance of the proximate or immediate causes of per-

* Communicated by the Author.

$\dagger$ See Edinburgh Journal of Science, First Series, vol. v. p. 52.

Third Series. Vol. 1. No. 4. Oct. $1832 . \quad 2 \mathrm{~K}$ 


\section{Mr. T. Smith's Investigation of certain Phenomena of}

ception in general, the uncertain state of our knowledge of any function of sight beyond the mere formation of the image on the retina, will, I trust, be deemed an apology for the minuteness of research and detail into which I have entered. My wish has been to advance nothing which is not founded on accurate observation, or experiment and legitimate induction.

In entering upon the investigation, it is of much importance to form a correct conception of the optical relations of the eyes at the time of making the experiments, and of the several objects concerned in them. For this purpose, and to make the phænomena themselves better understood, a diagram will

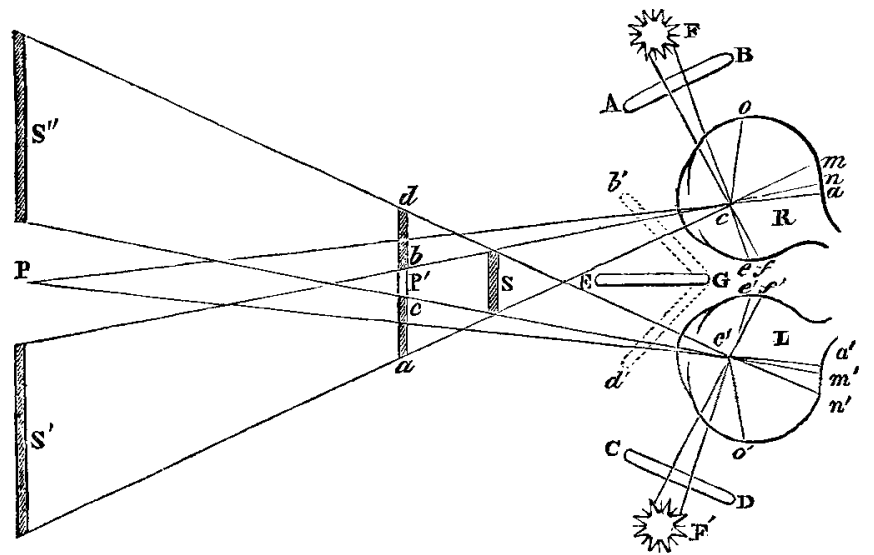

be useful. Let $R$ and $L$, represent the right and left eyes; $\mathbf{P}$ a distant point to which they are both directed; $\mathbf{S}$ a narrow slip of white paper placed vertically between that point and the eyes, and about ten or twelve inches from the latter: $F$ the flame of a lamp or candle near the right eye, and so placed that rays from it may enter that eye freely, while they are excluded from the left by an intervening obstacle $\mathbf{E} \mathbf{G}$; and $\mathrm{F}^{\prime}$ an equal flame placed near the left eye.

By the principles of optics, we know that straight lines drawn from the objects through the optical centres of the eyes, viz. $c$ and $c^{\prime}$, will touch those parts of the retina in each eye on which the several images fall. Hence $e f$ represent the place of the image of $\mathrm{F}$ in the right eye; $e^{\prime} f^{\prime}$ that of the image of $\mathrm{F}^{\prime}$ in the left; $m n$ and $m^{\prime} n^{\prime}$ the places of the images of $\mathbf{S}$; and $o$ and $\sigma^{\prime}$ the parts where the light from $F$ and $F^{\prime}$ would strike, if not prevented by the screen $\mathbf{E}$.

It is a law of vision, that objects are perceived in the direc- 
tions of right lines drawn from their images on the retina through a certain point which has been termed the centre of visible direction. Hence supposing $c$ and $c^{\prime}$ to be these points in the two eyes, the slip of paper $S$, by the operation of this law, will be perceived by the right eye somewhere between the lines $m c, n c$ produced, and by the left eye somewhere between $m^{\prime} c^{\prime}, n^{\prime} c^{\prime}$ produced. If $\mathrm{S}$ appears at the distance of the point in which the axes of the two eyes meet, then two images $\mathbf{S}^{\prime}$ and $\mathbf{S}^{\prime \prime}$ will be seen when the eyes are directed to $\mathbf{P}$; the two images will overlap each other when the axes meet in $\mathbf{P}^{\prime}$, and they will coalesce into one object when the eyes are directed to $\mathbf{S}$.

These things being premised, we shall now proceed to state, as briefly as possible, the experiments by which the phænomena that require particular attention may be exhibited.

Exp. 1. The two eyes being equally sensible to light, and the two lights $F$ and $F^{\prime}$ being placed as in the figure, make the slip of white paper $S$ appear double, by directing both eyes to a distant point $P$. Between one of the eyes $L$ and the light next it interpose the screen $\mathrm{CD}$, the right eye alone being exposed to the direct light $\mathrm{F}$. As soon as this unequal exposure of the two eyes is made, the two images of $S$ will be observed to change their colour; $S^{\prime}$, which is seen hy the exposed eye, becoming green, and $\mathrm{S}^{\prime \prime}$, which is seen by the shaded eye, becoming red. Withdraw the screen $\mathrm{CD}$ and interpose the screen $A B$; when $L$, which is now the exposed eye, will see the image $S^{\prime \prime}$ change from red to grcen; while $\mathbf{S}^{\prime}$, which is now the image seen by the shaded eye, will change from green to red. 'The same phænomena occur, and are even more vivid, if one of the eyes is exposed to the rays of the sun either direct or reflected.

Exp. 2. In the first experiment the images of $S$ fall on the retina out of the axes of the eyes $a$ and $a^{\prime}$. If this circumstance should be suspected to have any part in producing the green and red appearances, direct the two eyes to $S$, thus bringing the two images $m n, m^{\prime} n^{\prime}$ into the axes. When $\mathrm{S}$ is seen by both eyes at once, in this case it appears of its proper white colour; but if the screen $\mathrm{E} \mathrm{G}$ is turned alternately to the positions $\mathbf{G} b^{\prime}, \mathbf{G} d^{\prime}$, so as to make $\mathbf{S}$ visible to only one of the eyes at once, then it will be found to appear green to the exposed eye, and red to the shaded one.

This observation is an important one in another point of view, as it proves that the red and green colours of the two images are complementary to one another, a fact which is demonstrated otherwise by directing the two eyes to $\mathrm{P}^{\prime}$; for the part $b c$, where the two images overlap each other, appears white, though the rest of them $a c, b d$, appear red and green. $2 \mathrm{~K} 2$ 


\section{Mr. T. Smith's Investigation of certain Phcenomena of}

Exp. 3. When both eyes, directed to P, are shaded by means of the screens A B and C D, the two images $S^{\prime}$ and $\mathbf{S}^{\prime \prime}$ appear white. The same is true when both eyes are exposed by withdrawing the screens; but a difference in the degree of their whiteness occurs in these two circumstances, which is too important to pass unheeded. When both eyes are shaded, having attended well to the degree of whiteness which the two images of $\mathrm{S}$ assume, suddenly withdraw both of the screens; and though the two images will still seem white, yet they will appear sensibly darker than before, as if a thin cloud had been suddenly interposed between them and the source of their illumination. By interposing the screens again, their lustre is suddenly and permanently restored.

Exp. 4. To ascertain through what portion of the retina this extraordinary affection of sight occurs, I made the image of the slip of white paper $S$ traverse the retina of the exposed eye, from the borders of the bright inage $e f$ to $o$ and the corresponding parts of the shaded eye; but I could not observe that the green appearance to the exposed eye and red appearance to the shaded eye, was less at any one point than another. If the bright image fell on the centre of one retina, every part of the retina around that part, both in it and the other eye, appeared, as far as the image of $\mathbf{S}$ remained perceptible, under the operation of this affection.

These four experiments illustrate the principal facts that require to be kept in view. It is obvious that the green appearance of the white paper $S$ to the exposed eye, and the complementary red colour which it assumes to the other eye, are phænomena which admit of explanation in two ways; for they may be accounted for either by an existing excess of sensibility to the apparent colour, or by a defect of sensibility to its complementary colour. The first step, therefore, which I took in this stage of the inquiry into the causes of the phænomena, was, to investigate the precise nature of the effects produced by the action of the bright light $\mathrm{F}$ on one eye. For this purpose I made the following experiments.

Exp. 5. Having painted a narrow piece of white paper as nearly as I could of the green colour which the white slip S assumed to the exposed eye in the first experiment, I substituted it in the place of $\mathbf{S}$; and when my two eyes were directed to $P$, I observed that the left-hand image $S^{\prime}$, which was seen by the exposed eye, appeared of a deep green colour, and the right-hand image $\mathrm{S}^{\prime \prime}$, seen by the shaded eye, of a bright rohitish colour. When the two images were made to coalesce partially by directing the eyes to $\tilde{\mathrm{P}}^{\prime}$, the part $b c$, in which both colours united, appeared of that degree of green colour which I had given to $\mathrm{S}$. 
From the latter part of this, and also from the second experiment, an important piece of knowledge is derived; namely, that whatever be the state of the sensibility in the one eye, that state is exactly reversed at the same time in the other eye. Hence, if the deep green appearance of $\mathrm{S}^{\prime}$ in this last experiment is owing to an excess of the perception of the green light, the bright whitish appearance of $S^{\prime \prime}$ must be derived from a defective perception of the green light. We know that the green colour of the slip of paper used in this experiment was not owing to an absolute defect of the red and other primary coloured rays, but to an excess or predominance of the green light reflected from it. 'The whitish appearance of $\mathbf{S}^{\prime \prime}$, therefore, might be the result either of insensibility to this excess of green, or of an increase of sensibility to the light which is complementary to green. The whitish appearance, therefore, observed by the shaded eye in the fifth experiment is altogether equivocal, unless strict attention is paid to its degree, as will be manifest from the following considerations. White is the result of a mixture of any two antagonist or complementary colours in certain proportions. Let us, for the sake of illustration, suppose these proportions to be equal; then one part of red light and one part of complementary green, or two parts of each, or, in short, any equal parts of each, will form white, the brilliancy of which will be as the number of rays composing it. Now suppose a surface which appears green to contain one part of red and five of green, it is obvious that if this surface is converted to white by an addition of red light, it will be five times brighter than if it is made white by a removal of green light. The white colour of the green slip to the shaded eye, in the fifth experiment, appeared to me much brighter than could be accounted for by insensibility to the superabundant green light. But to remove all uncertainty on the subject, I had recourse to the following experiment.

Exp. 6. I laid on a deep black ground a slip, about one twentieth part of an inch in breadth, of the same green paper which $I$ had used in the last experiment, in a straight line with a slip of white paper of the same breadth. I viewed them both through a prism held parallel to their length, and observing that the red margin appeared much broader and brighter in the white than in the green slip, I shaded the white one with China ink, by degrees, until the red border in it appeared exactly like that of the green slip, expecting to obtain in this manner a very correct specimen of the degree of whiteness which the green slip used in the last experiment would assume, if the eye was insensible to the predominant green rays : that it was so, there cannot, on reflection, be any doubt. I therefore used this shaded slip as a test on repeating 


\section{Mr. T. Smith's Investigation of certain Phcenomena of}

the fifth experiment; and found that, compared with it, the image $S^{\prime \prime}$ seen by the shaded eye was incomparably brighter: whence it is quite certain that the sensibility is increased to $r e d$, and not impaired to green in the unexposed eye.

As the sensibility being increased to red light is the cause of the appearance to the shaded eye, it follows, from what has been remarked above, that the appearance to the exposed eye arises from equally diminished sensibility to the same colour. As this induction, however, may not appear so complete as it ought to be, on account of the phænomena mentioned under the third experiment, the state of the sensibility to red light in the exposed eye may be determined by direct experiment, thus:-

Exp. 7. Having found, by examination with the prism, that red morocco beather reflected few or none of the green rays when exposed to moderate white light, I put a slip of it in the position $S$, expecting that the image seen by the exposed eye would appear very dark or nearly black if the sensibility was remarkably diminished to red, but that it would appear bright wohite if the sensibility was increased to green: the result was, that it appeared a very dark red, and even (when the light $F$ was very brilliant) almost black.

Although these experiments appear to establish the truth of the conclusion, that the green and red appearances of the white surface $S$, in the first and second experiments, were owing to changes in the sensibility to, or perception of, red light alone; yet it is proper that we should pause here, to consider the fact disclosed by the third experiment, viz. that the two images of $\mathbf{S}$ appeared whiter when the two eyes were shaded from, than when they were both exposed to, the same quantity of bright light $\mathrm{F}$ and $\mathrm{F}^{\prime}$. The result in the third experiment may be thus expressed-diminished sensibility to rohite light by the equal action of bright light on both eyes. Now the results obtained by the first and second experiments have been proved to arise from the sensibility to red light being diminished in the exposed eye, and increased equally, as appeared, in the shaded eye: the effect, therefore, to be expected from exposing both eyes, is decreased, and equally increased seusibility to red light in each eye, or, in other words, an unchanged state of the perception of the white of both images of $\mathrm{S},-\mathrm{a}$ result manifestly at variance with that of the third experiment. But this was not the only perplexing circumstance encountered in this stage of the inquiry; for in repeating the experiments with white, red, and green slips of paper by turns, it could not long escape observation, that a partial coalescence of the two images, as at $b c$, gave a brighter colour than a total coalescence at $\mathrm{S}$. Happily, however, an 
affection of vision, which $I$ had discovered several years before, and even laid before the public (though in a manner that appears to me very unsatisfactory now), afforded a key to these difficulties.

It was commonly taught, and, if I am not mistaken, still continues to be so, that in any pre-existing state of the sensibility of the eye, the apparent luminousness of an object is in the direct ratio of the quantity of light from any point of it entering the eye and acting on any point of the retina. Physiologists had been led, by certain appearances, to adopt as a principle, that the sensibility of the retina is fatigued, impaired, or exhausted by the continued action of strong light; but no one had ever suspected that the sensibility could be augmented in consequence of the action of bright light, or that it could suffer a diminution without the action of light to account for it: yet I ascertained, by a series of unequivocal experiments, that, in certain circumstances, the action of a stronger and weaker light on adjoining parts of the retina was attended with an increased perception of the colour of the brighter object, and a diminished perception of the same colour in the darker one*. Attention to

* Some important errors having crept into the account which I formerly published of this affection of sight, I find it necessary, instead of referring to it, to explain here the facts by which the existence of the principle announced in the text appears to be demonstrated.

Take six pieces of thin white writing-paper, about four inches square each, and having painted them of the principal primary colours, viz. one red, another orange, a third yellow, a fourth blue, a fifth green, and the sixth violet, form them into tubes, to be used as will be directed immediately. Form also two other tubes of the same size,- - one of them of thin white writing-paper, the other opake and blackened within.

Now if one of the coloured tubes is applied to one of the eyes, the other eye being naked, and if the tube be strongly illuminated, a white surface, visible to both eyes, will present the following remarkable appearances. The round spot of the white surface that is seen through the tube will appear, not white, but uniformly of the colour that is complementary to that of the tube; while the rest of it will appear of its proper white colour to the naked eye. Thus if the tube employed is red, the circular spot seen through it will appear green; through the yellow tube it will appear purple; through the blue tube orange, \&c.; and the power of the colour of the tube to affect the perception of a white surface seen through it may. be still further illustrated by using tubes of different shades of the same primary colour. If two such tubes, differing only in degree, be applied to the two eyes at once, and directed to different parts of the white surface, the two circular spots thus seen will be as different in the degree of the complementary colour as the tubes are in the primary colour. If we attend to the optical state of the eye during these experiments, it will be found that every part of the retina, except a small circular spot towards the middle of it, is acted upon by the coloured light from the tube, and that this circular spot is acted upon by the white light from the surface seen through the tube. How is it, then, that we do not see the circular spot white, but of a colour which constantly varies so as to be complementary 


\section{Mr. T. Smith's Investigation of certain Phenomena of}

the second experiment soon convinced me that the action of the stronger lights $\mathbf{F}$ and $\mathbf{F}^{\prime}$, and of the weaker light from $\mathbf{S}$, and all surrounding objects, placed the retina in precisely the same condition in which the affection now mentioned had been developed in the experiments referred to. This principle, therefore, accounted for the two images of $S$ appearing darker when the eyes were exposed to, than when they

to that of the tube? M. Meusnier observed that, when the sun shone through a hole a quarter of an inch in diameter in a red curtain, the image of the luminous spot was green. M. Meusnier's retina in this case was evidently in a state closely analogous to that of the eye looking through a tube in any of the above instances. It has been proposed to explair the false perception in M. Meusnier's case on the principle, "that when the whole, or a great part, of the retina has the sensation of any primitive colour, a portion of the retina protected from the impression of the colour is actually thrown into that state which gives the accidental or harmonic colour." -(Vide Lardner's Cyclopædia, "Optics," p. 310.) If this principle exists, the appearance of the white surface seen through any of the coloured tubes would be owing to an inerease of sensizility to the complementary colour in that part of the retina where the white light acts ; but till direct proof of that principle is obtained, it cannot be denied that the phænomena would be equally well accounted for, by supposing the sensibility diminished to those rays of the white surface, which are of the same colour as the tube; for a white surface will appear as distinctly green by the abstraction of red as by the addition of green. To determine, therefore, to which of these causes the phænomena were due, I adopted the following plan.

As whiteness is the result of the united action of all the primary coloured rays, so a white tube may be regarded as an assemblage of all the primary coloured tubes into one. Hence, in viewing a white surface through a white tube, we ought to have a combination of all the effects that are obtained on-viewing the same white surface through the primary coloured tubes separately. Consequently, if the green appearance of a white surface seen through a red tube is owing to an increase of the sensibility to green, and so on of the others, then it is obvious that a union of all these increased sensibilities will constitute an increased sensibility to white; and, therefore, that a white surface, viewed through a white tube, ought to appear brighter than it does to the naked eye. But if the appearance of the white surface through each coloured tube is owing to diminished sensibility to those rays from the white surface that give the same colour as the tube, then a union of all the diminished sensibilities will form a diminished sensibility to white, consequently make the white surface seen through the white tube appear darker than it does to the naked eye. Experiment declares unequivocally in favour of the latter state of the sensibility; for the circular area of the white surface, which was seen through a white tube highly illuminated, appeared exceedingly dark, and if the illumination of the tube was very brilliant, almost black, compared with the other parts of it, which were seen of their ordinary white colour by the naked eye. In this manner, therefore, we are enabled to pronounce with certainty, that the false vision of the white surface seen through a coloured tube, is owing to the sensibility being diminished to the action of that kind of rays in the white image, which predominates in the tube employed.

By a proper management of the illumination of the white tube and white surface seen through it, we soon find that this diminished sensibility is only to a weaker light in the vicinity of a stronger; and further, that there 


\section{Vision, tracing them to Functional Actions of the Brain. 257}

were shaded from, the bright light, and thus reconciled the result of the second experiment to that of the others. But when the exciting cause of this affection (See Note*, p. 255, \&c.) is understood, when it is known that it occurs only when one of the objects is seen indistinctly from any cause (such as the image falling on the retina before or behind the focal point of the rays composing it) $\dagger$, all difficulty in regard to the brighter

always occurs at the same time an increase of the sensibility to the stronger light next to a weaker; for wher the white tube is strongly, and the white surface weakly, lighted, the appearances of the latter are always such as I have stated above, to the two eyes. If the lights are now pluced so as to make the illumination of the tube and surface more equal, the difference to the two eyes becomes less and less, till when both tube and surface are equally iluminated, the area seen through the tube appears as white as the rest of it does to the naked eye-a satisfactory proof, as appears to me, that the sensibility is only diminished to the weaker light in the vicinity of a stronger. But if the lights are so placed as to illuminate the white surface more than the tube, the former begins to appear brighter through the tube than to the naked eye; and when the difference of illumination is very great, as when we view a white surface through a black tube, the surface, which appears of its proper brightness to the naked eye, will appear over the circular part seen through the tube, as if the sun shone upon it and not upon the rest, - a decided proof that the sensibility is increased to the stronger light in the vicinity of a weaker. That the increase and decrease of sensibility here demonstrated occur at the same time and in the same degree, may be shown by carefully performing the following experiment. Take two pieces of paper, about four inches long and two inches broad each, the one bright white, the other painted dark, but not entirely black. Join the two pieces together so as to make one square piece, and at right angles to the line of their junction draw three straight lines with black ink through the white division, one of the lines being in the middle of it, and the other two at the distance of about three quarters of an inch from it on each side. Direct the two eyes to any distant point, and without altering their direction, introduce the square piece of paper between the eyes and the point they are directed to, holding the line of junction of the white and dark parts horizontally, and so that the two outer black lines shall appear united through their whole length with the middle black line. If strict attention is now paid to the appearance of the white and dark divisions, it will be found, if the experiment is properly performed, that the white portion will appear to terminate in the dark one by a very bright belt; while the dark portion will appear at the same time to terminate in the white one by a darker or black belt of the same breadth, and both of these belts will be seen to have evanescent edges at the sides where they disappear in their respective surfaces. These appearances are manifestly results of the same affection of sight that occurs in looking through coloured tubes, and show clearly that the increased sensibility to the greater light, and the diminished sensibility to the lesser, in the vicinity of each other, take place at the same time and in the same degree, the one being dependent upon the other.

$\uparrow$ If a white wafer or round piece of white paper is steadily viewed on the middle of an extensive red ground, about a foot from the eyes, the retina will evidently be in the same physical condition in regard to the light falling on it, as when a white surface is viewed through a red tube duly illuminated, except that in the latter case the line of junction between
Third Series. Vol. 1. No. 4. Oct. 1832.
$2 \mathrm{~L}$ 
appearance of the two images of $S$ when they coalesce partially, as at $b c$, than when they coalesce totally at $\mathrm{S}$, will vanish; for the image on the retina is distinct in the latter case, and indistinct in the former.

Allowance being made, therefore, for the effects on the sensibility produced by the action of a greater light in the vicinity of a lesser, the results in all the preceding experiments will be found to be in strict accordance with the following conclusion : viz. that the green appearance of a white object through all parts of the retina around the bright image in the exposed eye is owing to diminished sensibility to red light; and the red appearance of a white object through all the corresponding parts of the shaded eye is oring to an equal increase of the sensibility to the same kind of light.

[To be continued.]

he red and white images is rendered indistinct by the scattered red rays; whereas in the former, both the surfaces and their line of junction are perfectly distinct : but when the eyes are fixed steadily on the centre of the wafer, it never fails to be seen of its proper white colour as long as it is seen without any dazzling or fatigue of the eyes. I fell into an important error in my first experiments on this subject, and thus was led, in my first publication adverted to in the text, to a misstatement of this fact, having too hastily averred that these changes in the sensibility took place in every case in which a greater and a lesser light were viewed together. I had no suspicion that indistinctness of vision could have anything to do in producing the changes; and therefore when, on fixing my eyes on the centre of a white circle on an extensive red ground till they became dazzled and fatigued, I saw the white circle appear green, I concluded, erroneously indeed, but perhaps not unnaturally, that the change in the sensibility from which the green appearance arose, had actually commenced the moment I fixed my eyes on the white circle. So convinced, indeed, was I, for a long time, of the correctness of this conclusion, that I was not a little surprised and disappointed, on submitting my observations to several friends, to find that though they unanimously agreed with me respecting the appearances of a white surface viewed through coloured tubes, yet not one of them could be got, without prompting, to declare that a white circle on a wide coloured ground presented the same appearances. At length, on finding that I myself never experienced the changes in the sensibility but when the object became indistinct, I was led to attend to the importance of that condition, which enabled me to reconcile my own experience with that of intelligent persons who formerly differed from me. The power of indistinctness to excite this affection is well illustrated by the effects of twilight, which is well known to throw a shade of indistinctness over objects. I lately witnessed a very striking instance of this. Some white and pink-coloured paper were lying together on my table one evening, when the light of day was just enough to show the colour of the red paper. One of the company present said, pointing to the white paper, What a beautiful green colour that is! and every person present agreed that it was so, though $I$ assured them it was actually white : and not a little surprise was excited when it was distinctly perceived to be so, after $I$ had removed the pink paper. A white image, reflected by coloured glass, appearing of its complementary colour, is also a fine instance of this effect of indistinctness. 